Research, Society and Development, v. 9, n. 2, e49922031, 2020

(CC BY 4.0) | ISSN 2525-3409 | DOI: http://dx.doi.org/10.33448/rsd-v9i2.2031

\title{
Similaridades entre normas ISO que abordam sistemas de gestão
}

\section{Similarities between ISO standards that address management systems}

Similitudes entre las normas ISO que abordan los sistemas de gestion

Recebido: 06/11/2019 | Revisado: 07/11/2019 | Aceito: 09/11/2019 | Publicado: 12/11/2019

\section{Diogo Pedriali}

ORCID: https://orcid.org/0000-0002-4579-2393

Centro Estadual de Educação Tecnológica Paula Souza, Brasil

E-mail: diogo.pedriali@hotmail.com

Marilia Macorin de Azevedo

ORCID: https://orcid.org/0000-0003-0225-8155

Centro Estadual de Educação Tecnológica Paula Souza, Brasil

E-mail: marilia.azevedo@fatec.sp.gov.br

Carlos Hideo Arima

ORCID: https://orcid.org/0000-0001-7922-0943

Centro Estadual de Educação Tecnológica Paula Souza, Brasil

E-mail: charima@uol.com.br

José Manoel Souza das Neves

ORCID: https://orcid.org/0000-0002-7277-9434

Centro Estadual de Educação Tecnológica Paula Souza, Brasil

E-mail: jmneves.fatec@gmail.com

\section{Resumo}

O objetivo deste artigo é identificar as similaridades entre normas ISO de sistemas de gestão com a intenção de direcionamento para integração de sistemas de gerenciamento, por meio da utilização de computação cognitiva e revisão da literatura científica internacional. Foram consultadas as bases de dados Google Acadêmico e CORE, e o período pesquisado foi de 2015 a 2019. Trata-se de uma pesquisa do tipo exploratória longitudinal e descritiva que se utilizou de revisão da literatura técnica-científica e de computação cognitiva. Foram selecionadas 56 publicações que abordam sistemas de gestão ISO, em concordância com os termos: padronização, similaridade e integração. Observa-se que há oportunidade de estudos para desenvolvimento de framework para sistemas integrados de gestão, normatização e integração de certificação de sistemas de gestão. 
Palavras-chave: Sistema Integrado de Gestão; ISO; Computação Cognitiva; Gestão Integrada.

\begin{abstract}
The objective of this paper is to identify similarities between ISO management systems standards with the intention of management systems integration using the cognitive computing and review of international scientific literature. The Google Scholar and CORE databases were consulted, and the research period was from 2015 to 2019. This is a longitudinal, exploratory and descriptive research that used a review of technical and scientific literature and cognitive computing. Fifty-six publications addressing ISO management systems were selected, in accordance with the terms: standardization, similarity and integration. There is opportunity for studies to develop framework for integrated management systems, standardization and integration of management systems certification.
\end{abstract}

Keywords: Integrated Management System; ISO; Cognitive Computing; Integrated Management.

\title{
Resumen
}

El objetivo de este trabajo es identificar las similitudes entre los estándares de los sistemas de gestión ISO con la intención de dirigir la integración de los sistemas de gestión mediante el uso de la computación cognitiva y la revisión de la literatura científica internacional. Se consultaron las bases de datos Google Scholar y CORE, y el período de investigación fue de 2015 a 2019. Esta es una investigación exploratoria longitudinal y descriptiva que utilizó una revisión de la literatura técnico-científica y la computación cognitiva. Se seleccionaron 56 publicaciones sobre sistemas de gestión ISO, de acuerdo con los términos: estandarización, similitud e integración. Se observa que los estudios tienen la oportunidad de desarrollar un marco para la integración de sistemas integrados de gestión, estandarización y certificación de sistemas de gestión.

Palabras clave: Sistema Integrado de Gestión; ISO; Computación Cognitiva; Gestión Integrada.

\section{Introdução}

Desde 1940, período marcado pela Segunda Guerra Mundial, as empresas de todos os setores produtivos buscam produzir mais utilizando o mínimo de recursos possíveis. O 
agrupamento de técnicas e ferramentas administrativas, tais como Manufatura Enxuta (Lean Manufacturing), Lean Seis Sigma, Balanced Scorecard (BSC), Sistema de Produção Toyota, entre outros, proporcionam oportunidades de se aumentar a produtividade, reduzir retrabalhos e desperdícios, melhorar o desempenho de equipes de trabalhos, melhorar processos e aumentar a lucratividade.

Como oportunidade de melhoria e redução de desperdícios, há oportunidade de integração de normas certificáveis que abordam requisitos que orientam a organização, instituição e administração de sistemas de gestão.

Observa-se que empresas que buscam diferencial no mercado, implantam sistemas de gestão certificados, tornando-se reconhecidas internacionalmente uma vez que passam por processos sistêmicos de auditoria e validação.

As empresas que adotam a certificação de seus sistemas de gestão, buscam o atendimento de requisitos definidos por clientes ou até mesmo adotam a certificação de seus sistemas de gestão de modo pró ativo, pois acreditam que há benefícios interessantes para a organização das rotinas da empresa.

As empresas adotam inicialmente a composição de equipes de trabalho separadas em departamentos, onde cada uma destas administram os requisitos de cada um dos sistemas de gestão. Exemplo comum observado, são empresas que detêm a certificação de seus sistemas de gestão da Qualidade, Meio Ambiente e Saúde e Segurança Ocupacional e que para a gestão de cada um destes sistemas instituem uma equipe de profissionais especializados que atuam unicamente no sistema para o qual foi direcionado.

Esta departamentalização resulta inicialmente, numa demanda de maior quantidade de recursos humanos para suporte aos sistemas de gestão definidos como importantes pela empresa e pode resultar em falhas de comunicação entre os departamentos uma vez que os focos são diferentes em cada sistema de gestão.

Observada esta ocorrência em múltiplas empresas de diversos setores da economia, surgem as perguntas: Quais são as similaridades entre as normas ISO que abordam requisitos de sistema de gestão? e também, quais os tópicos de cada norma ISO que apresentam oportunidade de integração?

O objetivo geral deste trabalho é identificar as similaridades entre as normas ISO de sistemas de gestão com a intenção de direcionamento para integração de sistemas de gerenciamento.

Este artigo tem como foco o estudo das similaridades entre as normas ISO certificáveis que abordam requisitos de sistemas de gestão, por meio de revisão bibliográfica 
auxiliada por computação cognitiva e também mediante a análise de documentos científicos publicados pelos autores do presente trabalho.

A coleta e análise de dados neste estudo se limitou às bases de dados CORE (core.ac.uk) e Google Acadêmico e somente os documentos científicos que tratam da integração de sistemas de gestão foram utilizados.

Este trabalho contém além da introdução, o referencial teórico que apresenta a estrutura conceitual sobre a temática; o método utilizado para o desenvolvimento do artigo, que está contido na terceira seção; na quarta seção são apresentados os resultados e as discussões pertinentes a síntese dos documentos coletados; as considerações finais, bem como a identificação de limitações e oportunidades de estudos futuros são apresentadas na quinta seção deste trabalho.

\section{Sistema de Gestão}

Sistema de gestão pode ser definido como o conjunto de elementos inter-relacionados ou interativos de uma organização para estabelecer políticas, objetivos e processos para alcançar esses objetivos (ABNT NBR ISO 9000, 2015).

Segundo a ISO (2019), um sistema de gestão é o modo pelo qual uma organização administra as partes, setores ou departamentos que constituem seu negócio, à fim de atingir os objetivos estrategicamente definidos

Os objetivos definidos pelas empresas podem estar relacionados a tópicos diferentes, tais como qualidade de produto ou serviço, eficiência operacional, desempenho ambiental, saúde e segurança ocupacional, segurança das informações, sustentabilidade empresarial, responsabilidade social, entre outros.

A complexidade do sistema de gestão acompanha o contexto de cada empresa bem como seu tamanho. Para pequenas empresas pode-se reduzir a quantidade de documentos orientadores, enquanto para grandes empresas, em especial as altamente regulamentadas, podem precisar de documentação e controles extensivos para cumprimento de suas obrigações legais (ISO.ORG, 2019).

A Organização Internacional de Padronização (ISO) é uma organização não governamental independente, constituída por 164 países, com sede na cidade de Genebra, Suíça, e que reúne especialistas para compartilhar conhecimento e desenvolver Normas Internacionais voluntárias, baseadas em consenso e relevância para o mercado global (ISO.ORG, 2019). 
Segundo a ISO (2019), as normas internacionais existem para "fazer as coisas funcionarem", e fornecem especificações de classe mundial para produtos, serviços e sistemas, para garantir qualidade, segurança e eficiência; e são fundamentais para facilitar o comércio internacional.

As normas internacionais da ISO garantem que produtos e serviços sejam seguros, confiáveis e de boa qualidade. Para os negócios, são ferramentas estratégicas que reduzem custos, minimizando desperdícios e erros e aumentando a produtividade. Estas normas ajudam as empresas a acessar novos mercados, nivelar as condições de concorrência para os países em desenvolvimento e facilitar o comércio global livre e justo (ISO.ORG, 2019).

Atualmente a ISO disponibiliza listagem atualizada das normas de sistema de gestão e faz a categorização destas entre norma de sistema de gestão Tipo A e norma de sistema de gestão Tipo B.

As normas de sistemas de gestão Tipo A possuem requisitos e diretrizes o que possibilita que uma empresa possa reivindicar certificação de conformidade, enquanto as normas de sistemas de gestão Tipo B possuem apenas recomendações ou informações de suporte, não sendo possível a solicitação de certificação de conformidade (ISO.ORG, 2019).

Neste artigo, os autores analisam a similaridade entre as normas: ISO 9001:2015 sistemas de gestão da qualidade; ISO 14001:2015 - sistemas de gestão ambiental; ISO 26000:2010 - responsabilidade social, ISO/IEC 27001:2013 - sistemas de gestão de segurança da informação, ISO 31000:2018 - gestão de riscos e ISO 45001:2018 - sistemas de gestão de segurança e saúde ocupacional.

A escolha por estas normas se deu devida consulta a lista de normas de sistemas de gestão mais comuns e gerais, disponibilizada pela ISO, e atualizada em 17 de outubro de 2019, onde que as normas ISO 9001:2015, ISO 14001:2015, ISO/IEC 27001:2013 e ISO 45001:2018 são normas que possuem Estrutura de Alto Nível (HSL) e são normas de gestão Tipo A, ou seja, passíveis de certificação. Já as normas ISO 26000:2010 e ISO 31000:2018 são normas de diretrizes e de uso voluntário e não são apropriadas a fins de certificação, porém os autores entendem que possuem valiosa importância para a constituição de uma norma ou um sistema integrado de gestão.

\subsection{Normas de Sistemas Integrados de Gestão}

Ainda não existe uma norma ISO reconhecida internacionalmente que proporcione a integração de diversos sistemas de gestão de requisitos. Mesmo a ISO tendo criado a HSL 
com o objetivo de possibilitar integração de partes de normas de sistemas de gestão pelas empresas, somente o manual ISO (2018), foi publicado até o momento.

Os autores Domingues, Fonseca, Sampaio e Arezes (2016), citam que após as edições ISO 9001:2015 e ISO 14001:2015, a ISO decidiu que todos os padrões de sistemas de gestão deveriam seguir uma estrutura semelhante "a Estrutura de Alto Nível”, e portanto os autores acreditam que essa harmonização favorece a implementação de sistemas integrados de gestão.

Nowicki, Kafel e Sikora (2013), citam as normas: Global SAI.AS/NZS 4581:1999 (Austrália e Nova Zelândia), HB 10190:2001 (Reino Unido), PAS 99:2012 (Reino Unido), NTS:1996 (Noruega), DS 8001:2005 (Dinamarca) e UNE 66177:2005 (Espanha), como normas que buscam oportunizar a integração de sistemas de gestão. Estes autores não evidenciam a existência de norma ISO que busca abordar a integração de sistemas de gestão.

\subsection{Sistema de Gestão da Qualidade}

Uma empresa que possui foco em qualidade apresenta comportamentos, atitudes, atividades e processos que agregam valor através da satisfação das necessidades e expectativas dos clientes. A qualidade dos produtos e serviços inclui também o valor percebido e o benefício para o cliente (ABNT NBR ISO 9000, 2015).

O sistema de gestão da qualidade possui atividades que proporcionam a identificação de objetivos e determina os processos e recursos necessários para alcançar os resultados desejados. Por meio do sistema de gestão da qualidade a Alta Direção pode dimensionar adequadamente a utilização dos recursos considerando as consequências de sua decisão a longo e curto prazo e também provê meios para identificação de ações para tratar consequências pretendidas e não pretendidas na provisão de produtos e serviços (ABNT NBR ISO 9000, 2015).

\subsection{Sistema de Gestão Ambiental}

De acordo com a norma ABNT NBR ISO 14001 (2015), o objetivo de um sistema de gestão ambiental é prover estrutura para a proteção do meio ambiente e possibilitar uma resposta às mudanças das condições ambientais em equilíbrio com as necessidades socioeconômicas. Um sistema de gestão ambiental proporciona o gerenciamento dos aspectos ambientais, cumprimento de requisitos legais e outros requisitos e abordagem de riscos e oportunidades para a empresa que o adota.

Uma abordagem sistemática para a gestão ambiental pode resultar num desenvolvimento sustentável, por meio de proteção do meio ambiente pela prevenção ou 
mitigação dos impactos ambientais adversos; mitigação de potenciais efeitos adversos das condições ambientais na organização; aumento do desempenho ambiental; controle ou influência no modo em que os produtos e serviços da organização são projetados, fabricados, distribuídos, consumidos e descartados, utilizando uma perspectiva de ciclo de vida que possa prevenir o deslocamento involuntário dos impactos ambientais dentro do ciclo de vida; comunicação de informações ambientais para as partes interessadas pertinentes (ABNT NBR ISO 14001, 2015).

\subsection{Sistema de Gestão de Saúde e Segurança Ocupacional}

É citado na norma ISO 45001 (2018), que a empresa é responsável pela saúde e segurança ocupacional dos trabalhadores que podem ser afetados por suas atividades, incluindo a saúde física e mental dos colaboradores.

O objetivo de um sistema de gestão de saúde e segurança ocupacional é fornecer estrutura para gerenciar os riscos e oportunidades de saúde e segurança ocupacional, por meio da prevenção de lesões e problemas de saúde relacionados ao trabalho. É importante para as organização que adotam este sistema de gestão eliminar os perigos e minimizar os riscos de saúde e segurança ocupacional, por meio de medidas preventivas e de proteção efetivas (ISO 45001, 2018).

\subsection{Responsabilidade Social}

Empresas em todo o mundo tornam-se cada vez mais cientes da necessidade e dos benefícios do comportamento socialmente responsável. O objetivo da responsabilidade social é contribuir para o desenvolvimento sustentável (ABNT NBR ISO 26000, 2010).

Ainda segundo a norma ABNT NBR ISO 26000 (2010), o desempenho de uma empresa em relação à sociedade em que opera e ao seu impacto no meio ambiente se tornou uma parte crucial na avaliação de seu desempenho geral e de sua capacidade de continuar a operar de forma eficaz. A percepção e a realidade do desempenho em responsabilidade social de uma empresa podem influenciar, em sua vantagem competitiva; sua reputação; sua capacidade de atrair e manter trabalhadores, conselheiros, sócios, acionistas, clientes ou usuários; na manutenção da moral, do compromisso e da produtividade dos empregados; na percepção de investidores, proprietários, doadores, patrocinadores e da comunidade financeira; e sua relação com empresas, governos, mídia, fornecedores, organizações pares, clientes e a comunidade em que opera. 
(CC BY 4.0) | ISSN 2525-3409 | DOI: http://dx.doi.org/10.33448/rsd-v9i2.2031

\subsection{Sistema de Gestão da Segurança da Informação}

Empresas de diversos setores e tamanhos: coletam, processam, armazenam e transmitem informações; reconhecem que informações e processos, sistemas, redes e pessoas relacionados são ativos importantes para alcançar os objetivos da organização; enfrentam uma série de riscos que podem afetar o funcionamento dos ativos; e abordam sua exposição percebida ao risco, implementando controles de segurança da informação. Todas as informações mantidas e processadas por uma organização estão sujeitas a ameaças de ataques, de erros, de incidentes naturais, tais como inundação ou incêndio, e estão sujeitas a vulnerabilidades inerentes ao seu uso (ISO/IEC 27000, 2018).

De acordo com a norma ISO/IEC 27000 (2018), um sistema de gestão de segurança da informação propõe abordagem sistemática para estabelecer, implementar, operar, monitorar, revisar, manter e melhorar a segurança das informações de uma empresa para atingir os objetivos de negócios.

\subsection{Gestão de Riscos}

Conforme citado na norma ABNT NBR ISO 31000 (2018), o objetivo da gestão de riscos é a criação e a proteção de valor; busca melhorar o desempenho, encorajar a inovação e apoiar o alcance de objetivos definidos pelas empresas que a realizam. O propósito da estrutura da gestão de riscos é apoiar a organização na integração da gestão de riscos em atividades significativas. A eficácia da gestão de riscos dependerá da sua integração na governança e em todas as atividades da organização, incluindo a tomada de decisão.

A integração da gestão de riscos apoia-se em uma compreensão das estruturas e do contexto organizacional. Estruturas diferem, dependendo do propósito, metas e complexidade da organização. Integrar a gestão de riscos em uma organização é um processo dinâmico e iterativo, e convém que seja personalizado para as necessidades e para a cultura da organização (ABNT NBR ISO 31000, 2018).

\subsection{Estrutura de Alto Nível}

As normas de sistema de gestão ISO, após serem revisadas, possuem Estrutura de Alto Nível (HLS), e a HLS tem como conceito principal a utilização de mesma sequência de tópicos e onde possível, faz com que o uso de termilogia comum entre as normas dos principais sistemas de gestão torne-se possível. A criação da HLS buscou melhorar a coerência e a simplificação do uso das normas e também favorecer as empresas que buscam operar um único sistema de gestão, mesmo atendendo aos requisitos de duas ou mais normas 
de sistemas de gestão simultaneamente (ISO.ORG, 2019).

\section{Método}

Neste trabalho os métodos escolhidos para a coleta de informações foram a revisão bibliográfica automática, aqui denominada conforme Vroegindeweij e Carvalho (2019), de sistema de computação cognitiva e também a pesquisa bibliográfica nas bases de dados Google Acadêmico e CORE.

O escopo desta pesquisa é restrito aos documentos científicos que abordam a integração e similaridade entre requisitos presentes em normas de sistemas de gestão publicados pela ISO.

Mediante acesso a lista de normas comuns de sistemas de gestão disponibilizado no site iso.org, as normas analisadas pelos autores foram as apresentadas no Quadro 1.

Quadro 1 - Normas ISO analisadas pelos autores.

\begin{tabular}{clc} 
Norma & \multicolumn{1}{c}{ Título } & Revisão \\
\hline ISO 9001 & Sistemas de Gestão da Qualidade & 2015 \\
ISO 14001 & Sistemas de Gestão Ambiental & 2015 \\
ISO 26000 & Responsabilidade Social & 2010 \\
ISO/IEC 27001 & Sistemas de Gestão de Segurança da Informação & 2013 \\
ISO 31000 & Gestão de Riscos & 2018 \\
ISO 45001 & Sistemas de Gestão de Segurança e Saúde Ocupacional & 2018 \\
\hline
\end{tabular}

Fonte: Adaptado de Marinkovic (2019).

Foi escolhido o método de computação cognitiva, por se tratar de um método que proporciona a possibilidade de coleta automatizada de documentos científicos publicados em bases de dados; neste trabalho, a base escolhida foi a CORE e também a identificação automática dos principais trechos internos dos documentos científicos que atendem as perguntas de interesse da pesquisa; para tanto utilizou-se a plataforma online IBM Watson Discovery.

De acordo com Chatterjee e Debnath (2018), o IBM Watson Discovery é um sistema baseado em computação cognitiva. A computação cognitiva é uma técnica que é uma mistura 
de diferentes técnicas, como aprendizado de máquina, processamento de linguagem natural, inteligência artificial, interação humana, e raciocínio.

A interação humana é a maneira pela qual os computadores podem interagir com os humanos; pode-se dizer que o processamento da linguagem natural é um subconjunto da interação humana. O raciocínio é a parte em que o sistema realmente pensa como seres humanos e produz respostas para as perguntas. Portanto, o IBM Watson geral é composto dessas técnicas. O IBM Watson pode pensar como os seres humanos, pode aprender e, até mesmo fornecer respostas para perguntas realizadas por humanos (Chatterjee \& Debnath, 2018).

Manualmente escolheu-se realizar a pesquisa bibliográfica utilizando diretamente a máquina de pesquisa disponibilizado no site Google Acadêmico, para garantir a contemplação da pesquisa de todos os termos escolhidos por meio do método de pesquisa comum, diferentemente da pesquisa e identificação por meio de inteligência artificial.

A coleta automática de dados, a bibliometria e a revisão bibliográfica exploratória, ocorreram no período de setembro e outubro de 2019.

As bases de dados escolhidas foram a CORE, que reúne grande acervo de artigos de acesso livre; e o Google Acadêmico, que segundo Martín-Martín, Orduna-Malea, Thelwall e Delgado López-Cózar (2018), mostra-se como uma base de dados que reúne documentos científicos de uma grande quantidade de outras bases de dados, o que possibilita a ampliação da quantidade de artigos identificados que abordam a temática de interesse.

Os termos utilizados no primeiro nível de pesquisa foi management system e os termos de refinamento de pesquisa, estes no segundo nível de pesquisa foram similarities, integration e standard.

A restrição de pesquisa e aplicação de filtros de seleção de documentos se deram por meio das delimitações: somente ocorrência dos termos escolhidos no título dos documentos; documentos científicos em inglês; publicados entre 2015 e 2019; somente arquivos em PDF; e de acesso livre.

Foram utilizados métodos diferentes para a coleta de dados, o que reforça a característica de pesquisa de natureza exploratória, conforme Prodanov e Freitas (2013), e adotou-se abordagem documental e restrita aos documentos científicos de acesso livre provenientes de organizações internacionais, periódicos e eventos científicos, dissertações e teses.

Para identificação da literatura principal e também para fundamentação e delineamento do referencial teórico do estudo, foi realizada bibliometria, que buscou 
identificar a distribuição da frequência de publicações sobre a temática nos últimos cinco anos e a distribuição dos artigos mediante a ocorrências dos termos de filtro de segundo nível presentes nos títulos dos documentos coletados.

A produtividade dos autores foi identificada por meio do levantamento do Scopus $h$ index de cada autor; a identificação da relevância dos periódicos foi realizada por meio da consulta direta na máquina de pesquisa do site scimagojr.com; e a distribuição da frequência das publicações selecionadas foi levantada com auxílio do programa EndNote.

A coleta automática de dados, utilizada neste trabalho, foi realizada por meio do software R. Neste programa, um script de pesquisa automática foi utilizado, para tanto, cadastro prévio na base de dados CORE e na plataforma de inteligência artificial IBM Watson Discovery, foi realizado.

O script utilizado possibilita a pesquisa dos termos definidos pelo usuário, somente nos títulos dos documentos disponíveis na base de dados, no idioma inglês e no período de publicação de interesse. Possibilita a obtenção organizada e padronizada dos metadados de interesse do pesquisador e também a criação automática de planilha eletrônica com os metadados resultantes da pesquisa automática. Também há a automatização, após a geração da planilha de metadados, da obtenção dos documentos científicos encontrados na extensão PDF, por meio do download para pasta definida pelo pesquisador.

Após o agrupamento dos documentos coletados automaticamente, foram utilizados os softwares Mendeley (para geração de arquivo de metadados BIBTEX) e EndNote; este último para refinamento de filtro e extração dos dados bibliométricos.

Finalizado o processo de refinamento de seleção de documentos, os arquivos foram enviados para plataforma de inteligência artificial IBM Watson Discovery; plataforma esta que trabalha em nuvem. Nesta plataforma, por meio da computação cognitiva, automaticamente foi gerada análise de sentimento dos artigos selecionados e as palavras mais utilizadas para conceitualização teórica pelos autores dos documentos científicos escolhidos.

No ambiente de pesquisa do IBM Watson Discovery, consultas (queries) foram elaboradas para a identificação automática dos trechos mais importantes dos documentos de interesse deste estudo. Vale ressaltar que nesta identificação não se considerou apenas o retorno dado automaticamente pela máquina de inteligência artificial, havendo por meio de análise crítica dos autores a verificação e validação dos resultados apresentados pela máquina antes da construção deste artigo.

A utilização do sistema de computação cognitiva, que se configura como recurso automático foi intencional para a identificação rápida dos trechos mais relevantes de cada um 
(CC BY 4.0) | ISSN 2525-3409 | DOI: http://dx.doi.org/10.33448/rsd-v9i2.2031

dos documentos, de acordo com os termos escolhidos. Desta forma, identificou-se quais os documentos são relevantes e que então deveriam ser detalhadamente analisados pelos pesquisadores.

Além da utilização do método de computação cognitiva, inteligência artificial e programas de fichamento, foi também utilizado o programa Excel, para filtrar os metadados obtidos e identificar os artigos relevantes para este estudo.

Após a análise dos dados, realizou-se primeiramente a verificação da consistência do estudo, para buscar identificar o nível de atendimento do objetivo inicialmente definido e também a consolidação da síntese dos resultados evidenciados pelos autores dos documentos selecionados.

\section{Resultados e Discussão}

Após o levantamento dos dados bibliométricos nas coleções presentes nas bases de dados CORE e Google Acadêmico, foram selecionados 56 artigos contendo os termos da pesquisa. O resultado da aplicação de filtros e indicação automática de relevâncias dos documentos é apresentado na Tabela 1, onde são identificadas as quantidades de documentos selecionados sob o foco de cada um dos termos de pesquisa.

Tabela 1 - Quantidade de documentos localizados.

\begin{tabular}{lrcc} 
& CORE & $\begin{array}{c}\text { Google } \\
\text { Acadêmico }\end{array}$ & Seleção \\
\hline \multicolumn{1}{c}{ Termos } & \multicolumn{2}{c}{ Total } & $\mathbf{5 6}$ \\
& $\mathbf{1}^{\mathbf{0}}$ Nível & \\
ISO management system & 8655 & 454 & 19 \\
& $\mathbf{2}^{\mathbf{0}}$ Nível & & \\
similarities & 4440 & 1 & 7 \\
integration & 3986 & 55 & 20 \\
standard & 229 & 96 & 10 \\
\hline
\end{tabular}

Fonte: Resultado da pesquisa.

A distribuição anual das publicações selecionadas sobre o tema é apresentada na Figura 1. Observa-se que a maior quantidade de artigos selecionados sobre a temática se deu no ano de 2015 e a menor quantidade de artigos publicados é do ano de 2019, porém, ressalta- 
Research, Society and Development, v. 9, n. 2, e49922031, 2020

(CC BY 4.0) | ISSN 2525-3409 | DOI: http://dx.doi.org/10.33448/rsd-v9i2.2031

se que os dados deste estudo foram levantados entre os meses de setembro e outubro de 2019 e a quantidade de artigos publicados pode ser alterada.

Figura 1 - Distribuição de artigos coletados e selecionados por ano.

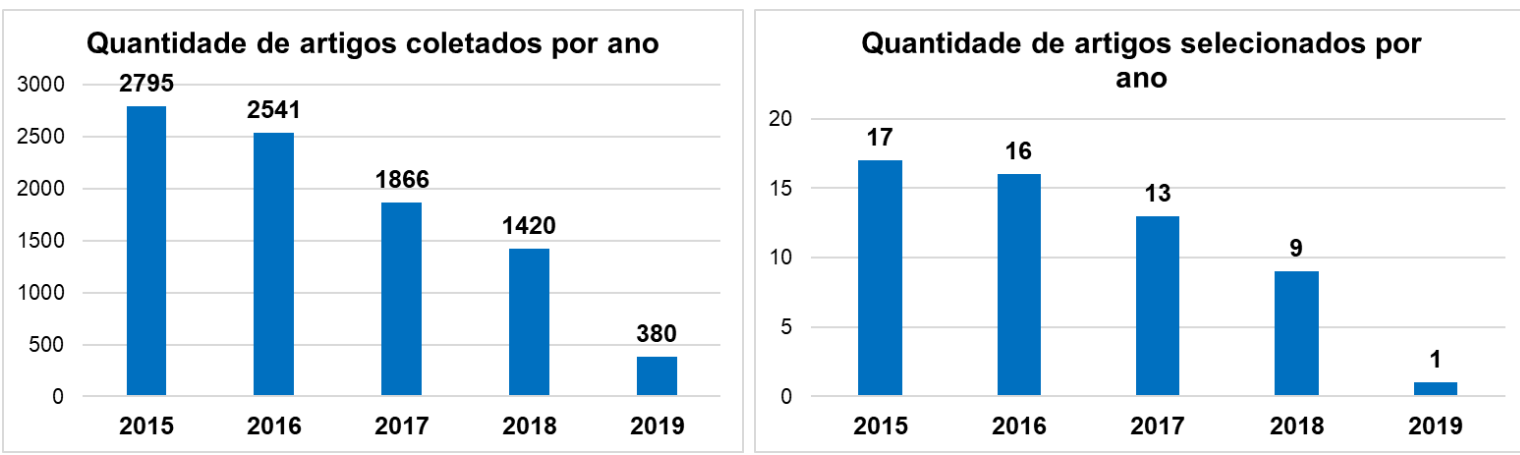

Fonte: Resultado da pesquisa.

A distribuição dos artigos coletados e filtrados em segundo nível por meio dos termos previamente especificados é representada na Figura 2. Observa-se que a maior quantidade de artigos encontrados possui em seus títulos os termos ISO management system e similarities.

Figura 2 - Quantidade de artigos filtrados em segundo nível.

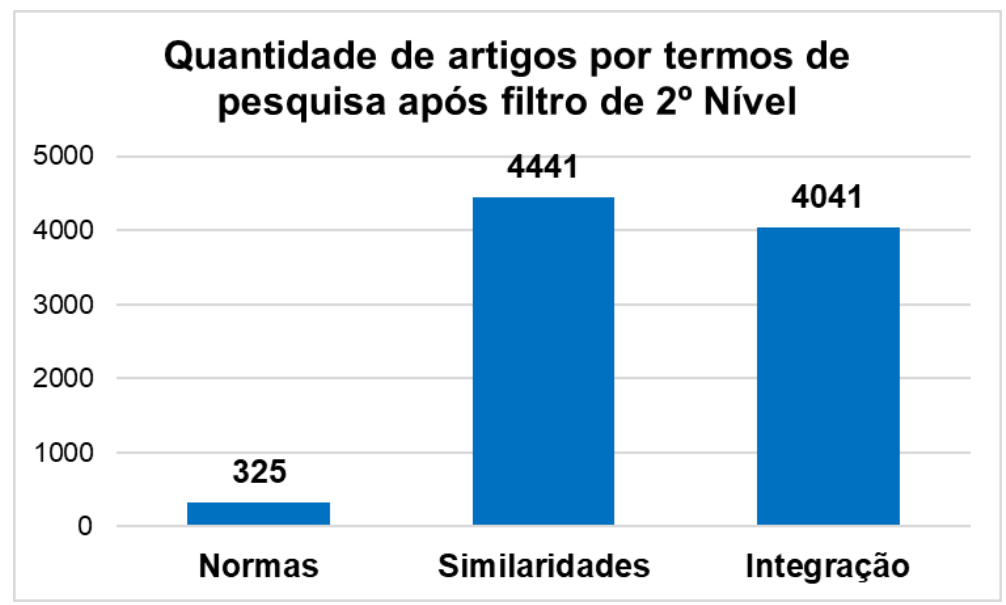

Fonte: Resultado da pesquisa.

Para validação da seleção automática de artigos por meio dos recursos de computação cognitiva, os autores realizaram a leitura do resumo de cada um dos artigos indicados via inteligência artificial para comprovar a correlação com a temática deste trabalho. Após consolidar a seleção de documentos, realizou-se a construção lógica e conceitual deste artigo.

Foram utilizados ao todo 90 documentos para a análise via computação cognitiva, considerando a seleção de documentos analisados pelos autores e também documentos que direcionam os conceitos, técnicas e fundamentos do próprio método de computação cognitiva. 
Research, Society and Development, v. 9, n. 2, e49922031, 2020

(CC BY 4.0) | ISSN 2525-3409 | DOI: http://dx.doi.org/10.33448/rsd-v9i2.2031

Observou-se que a análise de sentimento, que é realizada pela plataforma de computação cognitiva IBM Watson Discovery, o agrupamento de documentos utilizados pelos autores apresentam $88 \%$ de sentimentos identificados como positivos sobre o tema, $2 \%$ classificados com sentimento neutro e $10 \%$ classificados com sentimento negativo. Identificase que os documentos selecionados apresentam abordagens positivas sobre a temática da pesquisa.

Os termos conceituais identificados automaticamente pela plataforma de computação cognitiva foram: gerenciamento, gestão da qualidade, ISO 9000, sistema de gestão da qualidade e garantia da qualidade. Observa-se que nos documentos selecionados, a norma ISO 9001 é utilizada como orientadora para constituição de sistemas de gestão e acredita-se que isso se justifica por se tratar de uma das normas mais utilizadas para certificação e reconhecimento da qualidade das empresas no mundo, de acordo com o relatório da pesquisa Survey 2018, consolidado por Charlet (2018). Neste relatório é possível verificar que em 2018 haviam 878664 certificados ISO 9001:2015 válidos distribuídos em 1180965 organizações no mundo.

Na Figura 3 é possível evidenciar os certificados válidos em 2018 identificados na pesquisa survey realizada pela ISO. Importante ressaltar que as normas ISO 26000 e ISO 31000 não são normas certificáveis e, portanto, não constam nos resultados de certificados válidos no relatório da pesquisa.

Figura 3 - Quantidade de certificados identificados na pesquisa ISO Survey 2018.

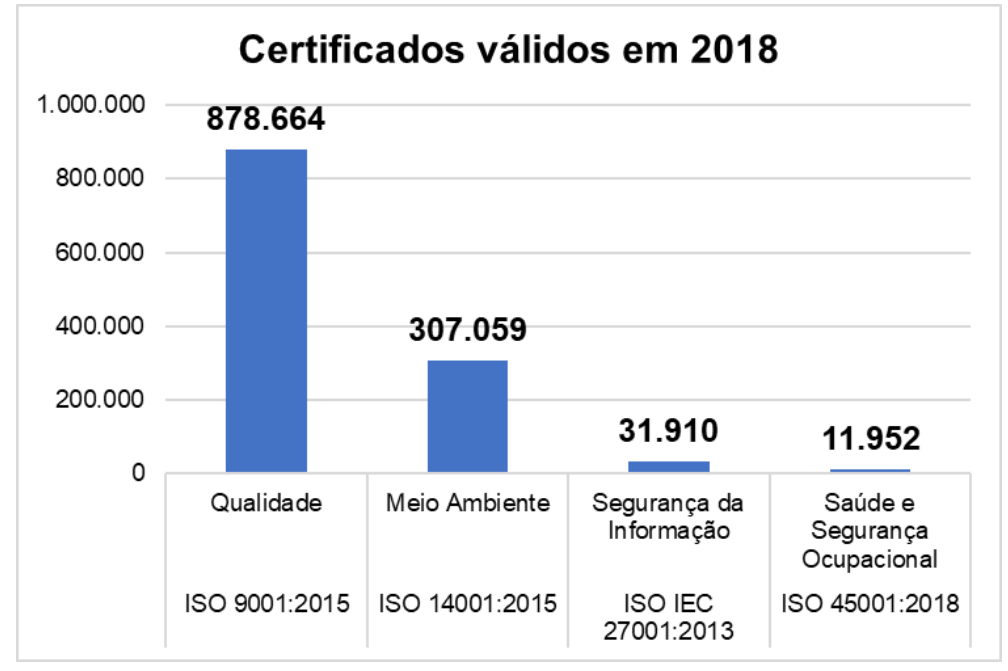

Fonte: Adaptado de Charlet (2018).

Os benefícios de um sistema de gestão eficaz pode resultar para a organização que o detêm, o uso mais eficiente dos recursos e melhor desempenho financeiro; melhor gerenciamento de riscos e proteção das pessoas e do meio ambiente, e maior capacidade de 
fornecer serviços e produtos consistentes e aprimorados, aumentando assim o valor para os clientes e todas as outras partes interessadas (ISO.ORG, 2019).

Torna-se interessante o agrupamento dos benefícios citados pelos especialistas participantes da constituição do agrupamento dos requisitos presentes nas normas ISO de sistemas de gestão.

Os benefícios evidenciados pelos autores, nas normas ABNT ISO 9000 (2015) e ABNT NBR ISO 9001 (2015) são: capacidade de prover consistentemente produtos e serviços que atendam aos requisitos do cliente e aos requisitos estatutários e regulamentares aplicáveis; facilitação de oportunidades para aumentar a satisfação do cliente; abordagem de riscos e oportunidades associados com seu contexto e objetivos; capacidade de demonstrar conformidade com requisitos especificados de sistemas de gestão da qualidade.

Na norma ABNT NBR ISO 14001 (2015), os benefícios citados foram: aumento do desempenho ambiental; atendimento dos requisitos legais e outros requisitos; alcance dos objetivos ambientais; proteção do meio ambiente pela prevenção ou mitigação dos impactos ambientais adversos; mitigação de potenciais efeitos adversos das condições ambientais na organização; auxílio à organização no atendimento aos requisitos legais e outros requisitos; aumento do desempenho ambiental; controle ou influência no modo em que os produtos e serviços da organização são projetados, fabricados, distribuídos, consumidos e descartados, utilizando uma perspectiva de ciclo de vida que possa prevenir o deslocamento involuntário dos impactos ambientais dentro do ciclo de vida; alcance dos benefícios financeiros e operacionais que podem resultar da implementação de alternativas ambientais que reforçam a posição da organização no mercado; comunicação de informações ambientais para as partes interessadas pertinentes.

Evidencia-se na norma ISO 45001 (2018) os seguintes benefícios: redução de acidentes e doenças ocupacionais; desenvolvimento de uma cultura prevencionista; maior conformidade com requisitos legais e outros; incorporação de boas práticas de gestão; melhor organização dos processos de trabalho; processo efetivo para identificação de perigos; controle de riscos; avaliação contínua do desempenho e monitoramento dos processos de saúde e segurança ocupacional.

Nas normas ISO/IEC 27000 (2018) e ABNT NBR ISO/IEC 27001 (2013), os benefícios são: preservação da confidencialidade, integridade e disponibilidade da informação por meio da aplicação de um processo de gestão de riscos; fornecimento de confiança para as partes interessadas de que os riscos são adequadamente gerenciados; assistência à gerência, consistentemente, gerenciando e operando de maneira responsável sua abordagem ao 
gerenciamento da segurança da informação, no contexto do gerenciamento e governança corporativa de riscos, incluindo a educação e treinamento de proprietários de empresas e sistemas no gerenciamento holístico da segurança da informação; promoção de boas práticas de segurança da informação aceitas globalmente, de maneira não prescritiva, dando às organizações a liberdade de adotar e aprimorar controles relevantes que atendam às suas circunstâncias específicas e mantê-las diante de mudanças internas e externas; fornecimento de uma linguagem comum e base conceitual para segurança da informação, facilitando a confiança dos parceiros de negócios; aumento da confiança das partes interessadas na organização; satisfação das necessidades e expectativas da sociedade; gestão econômica mais eficaz dos investimentos em segurança da informação.

Os benefícios citados na norma ABNT NBR ISO 26000 (2010) são: estímulo a um processo decisório com decisões fundamentadas e baseadas em uma melhor compreensão das expectativas da sociedade, das oportunidades associadas à responsabilidade social (inclusive um melhor controle dos riscos legais) e dos riscos de não ser socialmente responsável; melhoria das práticas de gestão de risco da organização; melhoria da reputação da organização e promoção de uma maior confiança por parte do público; suporte à licença de operação de uma organização; geração de inovação; melhoria da competitividade da organização, incluindo acesso a financiamento e status de parceiro preferencial; melhoria do relacionamento da organização com as suas partes interessadas, dessa forma expondo a organização a novas perspectivas e ao contato com diferentes partes interessadas; aumento da fidelidade, do envolvimento, da participação e da moral dos empregados; melhoria da saúde e segurança dos trabalhadores de ambos os sexos; impacto positivo na capacidade da organização de recrutar, motivar e reter seus empregados; economia resultante do aumento de produtividade e eficiência no uso dos recursos, redução no consumo de energia e água, redução do desperdício e recuperação de subprodutos valiosos; maior confiabilidade e equidade das transações por meio de envolvimento político responsável, concorrência leal e ausência de corrupção; prevenção ou redução de possíveis conflitos com consumidores referentes a produtos ou serviços.

São citados na norma ABNT NBR ISO 31000 (2018) os seguintes benefícios: aumento da probabilidade de atingir os objetivos; encorajamento de gestão proativa; prontidão para a necessidade de identificar e tratar os riscos através de toda a organização; melhoria da identificação de oportunidades e ameaças; atendimento das normas internacionais e requisitos legais e regulatórios pertinentes; melhoria do reporte das informações financeiras; melhoria da governança; melhoria da confiança das partes interessadas; estabelecimento de uma base 
(CC BY 4.0) | ISSN 2525-3409 | DOI: http://dx.doi.org/10.33448/rsd-v9i2.2031

confiável para a tomada de decisão e o planejamento; melhoria dos controles; alocação e utilização eficaz dos recursos para o tratamento de riscos; melhoria da eficácia e a eficiência operacional; melhoria do desempenho em saúde e segurança, bem como a proteção do meio ambiente; melhoria da prevenção de perdas e da gestão de incidentes; minimização de perdas; melhoria da aprendizagem organizacional; aumento da resiliência da organização.

Quando uma organização adota a gestão integrada de seus sistemas normatizados, observa-se conforme citado por Nowicki et al. (2013), diversos benefícios tais como: maior competitividade das empresas; melhorar a eficácia e eficiência da organização, evitando duplicação de trabalho; reduzir a burocracia, eliminando a duplicação de políticas, procedimentos e registros; harmonizar os objetivos, processos e recursos; redução de custos, por exemplo, reduzindo o número de auditorias internas e externas; disponibilidade de treinamento conjunto e melhor comunicação em todos os níveis de gerenciamento.

Mesmo que as normas descrevam práticas para diferentes aplicações e abordagens, é possível encontrar semelhanças em suas descrições, bem como um nível diferente de detalhes. Mediante este fato é possível sugerir que ao se identificar as semelhanças entre as normas selecionadas pode-se harmonizar e integrar dois ou mais sistemas de gestão (Pardo, Pino, \& Garcia, 2016).

Durante esta pesquisa, buscou-se a identificação das similaridades das normas estudadas e a identificação de similaridades se dá pela indicação numéricas dos tópicos das normas, como é apresentada no Quadro 2.

Quadro 2 - Similaridades identificadas entre as normas selecionadas.

Tópicos

\begin{tabular}{|c|c|c|c|c|c|c|}
\hline Descrição & ISO 9001 & $\begin{array}{c}\text { ISO } \\
14001\end{array}$ & $\begin{array}{c}\text { ISO/IEC } \\
27001\end{array}$ & $\begin{array}{c}\text { ISO } \\
45001\end{array}$ & $\begin{array}{c}\text { ISO } \\
26000\end{array}$ & $\begin{array}{c}\text { ISO } \\
\mathbf{3 1 0 0 0}\end{array}$ \\
\hline Contexto da organização & 4 & 4 & 4 & 4 & $\begin{array}{l}4,4.1 \\
4.2,4.4 \\
4.6,4.7 \\
\quad 4.8\end{array}$ & 4 \\
\hline $\begin{array}{l}\text { Entendendo a organização e } \\
\text { seu contexto }\end{array}$ & 4.1 & 4.1 & 4.1 & 4.1 & 4.3 & $\begin{array}{c}5,5.4 \\
5.4 .1\end{array}$ \\
\hline $\begin{array}{l}\text { Entendendo as necessidades } \\
\text { e expectativas de partes } \\
\text { interessadas }\end{array}$ & 4.2 & 4.2 & 4.2 & 4.2 & $\begin{array}{l}4.5,5 \\
5.1,5.2\end{array}$ & $\begin{array}{r}6.3 .3 \\
6.3 .4\end{array}$ \\
\hline $\begin{array}{l}\text { Determinando o escopo do } \\
\text { sistema de gestão }\end{array}$ & 4.3 & 4.3 & 4.3 & 4.3 & - & $\begin{array}{l}6.3 \\
6.3 .1 \\
6.3 .2\end{array}$ \\
\hline $\begin{array}{l}\text { Sistema de gestão e seus } \\
\text { processos }\end{array}$ & 4.4 & 4.4 & 4.4 & 4.4 & $\begin{array}{l}6.3,6.4 \\
6.5,6.6 \\
6.7,6.8\end{array}$ & 5.3 \\
\hline
\end{tabular}


(continuação)

\begin{tabular}{|c|c|c|c|c|c|c|}
\hline Liderança & 5 & 5 & 5 & 5 & $\begin{array}{c}6,6.1 \\
6.2\end{array}$ & - \\
\hline $\begin{array}{l}\text { Liderança e } \\
\text { comprometimento }\end{array}$ & 5.1 & 5.1 & 5.1 & 5.1 & 5.3 & $\begin{array}{l}5.2 \\
5.4 .2\end{array}$ \\
\hline Política & 5.2 & 5.2 & 5.2 & 5.2 & - & - \\
\hline $\begin{array}{l}\text { Papéis, responsabilidades e } \\
\text { autoridades organizacionais }\end{array}$ & 5.3 & 5.3 & 5.3 & $\begin{array}{l}5.3 \\
5.4\end{array}$ & $7,7.1$ & 5.4 .3 \\
\hline Planejamento & 6 & 6 & 6 & 6 & - & - \\
\hline $\begin{array}{l}\text { Ações para abordar } \\
\text { aspectos, riscos e } \\
\text { oportunidades }\end{array}$ & 6.1 & $\begin{array}{c}6.1, \\
6.1 .1, \\
6.1 .2, \\
6.1 .3, \\
6.1 .4\end{array}$ & $\begin{array}{l}6.1, \\
6.1 .1 \\
6.1 .2, \\
6.1 .3\end{array}$ & $\begin{array}{c}6.1, \\
6.1 .1, \\
6.1 .2, \\
6.1 .3, \\
6.1 .4\end{array}$ & - & $\begin{array}{c}6.4, \\
6.4 .1, \\
6.4 .2, \\
6.4 .3, \\
6.4 .4\end{array}$ \\
\hline $\begin{array}{l}\text { Objetivos e planejamento e } \\
\text { programas }\end{array}$ & $6.2,6.3$ & $\begin{array}{c}6.2 \\
6.2 .1 \\
6.2 .2\end{array}$ & 6.2 & $\begin{array}{c}6.2 \\
6.2 .1 \\
6.2 .2\end{array}$ & $\begin{array}{c}7.2,7.3 \\
7.4\end{array}$ & - \\
\hline Apoio & 7 & 7 & 7 & 7 & - & - \\
\hline Recursos & $\begin{array}{c}7.1,7.1 .1, \\
7.1 .2, \\
7.1 .3, \\
7.1 .4, \\
7.1 .5, \\
7.1 .5 .1 \\
7.1 .5 .2, \\
7.1 .6\end{array}$ & 7.1 & 7.1 & 7.1 & - & 5.4 .4 \\
\hline Competência & 7.2 & 7.2 & 7.2 & 7.2 & - & - \\
\hline Conscientização & 7.3 & 7.3 & 7.3 & 7.3 & - & - \\
\hline Comunicação & 7.4 & $\begin{array}{l}7.4, \\
7.4 .1 \\
7.4 .2 \\
7.4 .3\end{array}$ & 7.4 & $\begin{array}{l}7.4, \\
7.4 .1 \\
7.4 .2 \\
7.4 .3\end{array}$ & 7.5 & $\begin{array}{l}5.4 .5 \\
6.2 \\
6.7\end{array}$ \\
\hline Informação documentada & 7.5 & 7.5 & 7.5 & 7.5 & - & - \\
\hline Generalidades & 7.5 .1 & 7.5 .1 & 7.5 .1 & 7.5 .1 & - & - \\
\hline Criando e atualizando & 7.5 .2 & 7.5 .2 & 7.5 .2 & 7.5 .2 & - & - \\
\hline $\begin{array}{l}\text { Controle de informação } \\
\text { documentada }\end{array}$ & 7.5 .3 & 7.5 .3 & 7.5 .3 & 7.5 .3 & - & 6.7 \\
\hline Operação & 8 & 8 & 8 & 8 & - & $\begin{array}{c}5.5,6, \\
6.1 \\
6.5, \\
6.5 .1\end{array}$ \\
\hline $\begin{array}{l}\text { Planejamento e controle } \\
\text { operacionais }\end{array}$ & 8.1 & 8.1 & 8.1 & $\begin{array}{l}8.1, \\
8.1 .1, \\
8.1 .2, \\
8.1 .3, \\
8.1 .4\end{array}$ & - & $\begin{array}{c}6.5 .2 \\
6.5 .3\end{array}$ \\
\hline
\end{tabular}


(continuação)

\begin{tabular}{|c|c|c|c|c|c|c|}
\hline $\begin{array}{l}\text { Requisitos para produtos e } \\
\text { serviços, preparação e } \\
\text { resposta a emergências }\end{array}$ & $\begin{array}{c}8.2,8.2 .1, \\
8.2 .2, \\
8.2 .3, \\
8.2 .4,8.3, \\
8.3 .1, \\
8.3 .2, \\
8.3 .3, \\
8.3 .4, \\
8.3 .5, \\
8.3 .6\end{array}$ & 8.2 & - & 8.2 & - & - \\
\hline $\begin{array}{l}\text { Controle de processos, } \\
\text { produtos e serviços providos } \\
\text { externamente }\end{array}$ & $\begin{array}{l}8.4,8.4 .1 \\
8.4 .2 \\
8.4 .3\end{array}$ & - & $8.2,8.3$ & - & - & - \\
\hline $\begin{array}{l}\text { Produção e provisão de } \\
\text { serviço }\end{array}$ & $\begin{array}{c}8.5,8.5 .1 \\
8.5 .2, \\
8.5 .3, \\
8.5 .4 \\
8.5 .5 \\
8.5 .6,8.6 \\
8.7\end{array}$ & - & - & - & - & - \\
\hline Avaliação de desempenho & 9 & 9 & 9 & 9 & $\begin{array}{l}7.6 \\
7.7\end{array}$ & 5.6 \\
\hline $\begin{array}{l}\text { Monitoramento, medição, } \\
\text { análise e avaliação }\end{array}$ & 9.1 & 9.1 & 9.1 & 9.1 & - & 6.6 \\
\hline Generalidades & 9.1 .1 & 9.1 .1 & - & 9.1 .1 & - & - \\
\hline $\begin{array}{l}\text { Satisfação das partes } \\
\text { interessadas }\end{array}$ & 9.1 .2 & 9.1 .2 & - & - & $\begin{array}{l}7.6 \\
7.7\end{array}$ & - \\
\hline Análise e avaliação & 9.1 .3 & - & - & 9.1 .2 & $\begin{array}{l}7.6 \\
7.7\end{array}$ & - \\
\hline Auditoria interna & 9.2 & $\begin{array}{l}9.2 \\
9.2 .1 \\
9.2 .2\end{array}$ & 9.2 & $\begin{array}{l}9.2 \\
9.2 .1 \\
9.2 .2\end{array}$ & - & - \\
\hline Análise crítica pela direção & $\begin{array}{l}9.3,9.3 .1 \\
9.3 .2 \\
9.3 .3\end{array}$ & 9.3 & 9.3 & 9.3 & - & 6.6 \\
\hline Melhoria & $10,10.1$ & $\begin{array}{c}10 \\
10.1\end{array}$ & 10 & $\begin{array}{l}10, \\
10.1\end{array}$ & 7.8 & $\begin{array}{c}5.7,5 \\
7.1\end{array}$ \\
\hline $\begin{array}{l}\text { Não conformidade e ação } \\
\text { corretiva }\end{array}$ & 10.2 & 10.2 & 10.1 & 10.2 & - & - \\
\hline Melhoria contínua & 10.3 & 10.3 & 10.2 & 10.3 & - & 5.7 .2 \\
\hline
\end{tabular}

Fonte: Resultado da pesquisa.

Os tópicos de 0 (introdução), 1 (escopo), 2 (referência normativa) e 3 (termos e definições) das normas abordam de modo semelhante, os elementos introdutórios e gerais das normas, desta forma não foram adicionados no Erro! Fonte de referência não encontrada., pois os autores escolheram dar luz à análise dos requisitos específicos destas. 


\section{Considerações finais}

Obter aumento de lucros e utilizar os recursos de modo mais racional e eficiente continua sendo foco das empresas no mundo e os atuais trabalhos acadêmicos evidenciam que a unificação ou integração de dois ou mais sistemas de gestão pode fornecer oportunidades para se atingir benefícios, tais como, aumento de lucratividade de uma operação econômica.

Empresas que adotam o sistema integrado de gestão de requisitos apresentam grande potencialidade de atendimento aos requisitos legais e a sustentabilidade econômica, financeira, ambiental, social e demonstram domínio integral de suas ações internas e externas.

Evidenciou-se por meio dos documentos selecionados que não há na ISO, no momento de desenvolvimento desta pesquisa, a configuração de comitê de técnico de trabalho como o foco na criação de uma norma que proponha o agrupamento de diretrizes e requisitos passíveis de serem certificados e reconhecidos internacionalmente pela sociedade.

A possiblidade de certificação do sistema de gestão integrado pode resultar em benefícios para as empresas, tais como melhores direcionamentos das atividades, equipe multidisciplinar, redução de lacunas não atendidas devido os interstícios existentes entre as normas, transmissão mais consistente e unificada das informações para as classes administrativas operacional, tática e alta gestão.

Observou-se ao analisar as similaridades entre as normas selecionadas pelos autores deste trabalho que os tópicos de 1, 2 e 3 das normas abordam de modo semelhante, os elementos introdutórios das normas; os tópicos 4, 5, 6, 7, 8, 9 e 10 nas normas ISO passíveis de certificação se correlacionam, por meio da justificativa de terem sido revisados e adequados a estrutura de alto nível desenvolvida pela ISO, o que proporciona harmonia entre os tópicos e indica a possiblidade de planejamento da adoção da integração dos sistemas de gestão.

Procurou-se atender o objetivo do trabalho por meio de apresentação do agrupamento dos tópicos que apresentam similaridade de abordagem e de requisitos entre as normas ISO de sistema de gestão. Apresentou-se também os benefícios indicados nas normas escolhidas e indicados nos documentos científicos coletados.

As dificuldades do processo de unificação de sistemas de gestão não foram satisfatoriamente atendidas por meio dos documentos selecionados para este estudo e isso é indicado pela análise de sentimento realizada por meio da plataforma de computação cognitiva IBM Watson Discovery uma vez que o sentimento negativo sobre a similaridade e integração de sistemas de gestão apresentou-se $10 \%$ negativo, desta forma surge a 
(CC BY 4.0) | ISSN 2525-3409 | DOI: http://dx.doi.org/10.33448/rsd-v9i2.2031

oportunidade de futuro trabalho com o objetivo de identificação das dificuldades evidenciadas pelas empresas no processo de adoção, operação e melhoria de seu sistema integrado de gestão.

Quanto ao método escolhido de coleta e identificação automática de artigos e o sistema de computação cognitiva, mostra-se como método viável para o direcionamento inicial das nuances e especificidades acerca da pesquisa, principalmente pela possiblidade de identificação rápida e automática de trechos importantes de uma grande quantidade documentos científicos que podem ser escolhidos pelo pesquisador, porém, o sistema ainda demanda melhoria quanto ao refinamento e aumento da precisão na identificação de termos no contexto da temática escolhida.

A utilização da computação cognitiva atualmente não exclui a necessidade de análise e validação humana, bem como não irá substituir tão logo a arte de interpretação, sintetização e escrita de documentos técnico-científicos, porém, demonstra-se como uma ferramenta interessante para ganho de produtividade e redução do tempo de seleção de materiais para pesquisas que utilizam a revisão de literatura como método.

Identificam-se como oportunidades de futuros trabalhos, a realização de estudos empíricos e longitudinais que utilizem métodos estruturados de triangulação de dados, para validação dos requisitos presentes nas normas ISO para a unificação de sistemas de gestão e então identificar os fatores que resultarão em benefícios internos e externos. A empresa que a adotar, poderia identificar as dificuldades correlacionadas a esta adoção; quantificação financeira da adoção do sistema integrado de gestão; demandas operacionais e de treinamentos para a manutenção do sistema nesta configuração; desenvolvimento de frameworks para integração de sistemas de gestão ISO; identificação das razões da ausência de normatização de sistemas integrados.

\section{Referências}

Associação Brasileira de Normas Técnicas. (2013). ABNT NBR ISO/IEC 27001 - Tecnologia da informação - técnicas de segurança - sistemas de gestão da segurança da informação requisitos. Rio de Janeiro.

Associação Brasileira de Normas Técnicas. (2015). ABNT NBR ISO 14001 - Sistemas de gestão ambiental - requisitos com orientações para uso. Rio de Janeiro. 
Associação Brasileira de Normas Técnicas. (2010). ABNT NBR ISO 26000 - Diretrizes sobre responsabilidade social. Rio de Janeiro.

Associação Brasileira de Normas Técnicas. (2018). ABNT NBR ISO 31000 - Gestão de riscos - diretrizes. Rio de Janeiro.

Associação Brasileira de Normas Técnicas. (2015). ABNT NBR ISO 9000 - Sistemas de gestão da qualidade - fundamentos e vocabulário. Rio de Janeiro.

Associação Brasileira de Normas Técnicas. (2015). ABNT NBR ISO 9001 - Sistema de gestão da qualidade. Rio de Janeiro.

Charlet, L. (2018). The ISO Survey of Management System Standard Certifications 2018. Recuperado 20 de outubro de 2019, de http://www.iso.org/iso/iso_survey_executivesummary.pdf

Chatterjee, J., \& Debnath, T. (2018). Environmental Monitoring Using Sense HAT based on IBM Watson IoT Platform. International Research Journal of Engineering and Technology (IRJET), 5(7), 392-399. https://doi.org/10.13140/RG.2.2.30943.56484

Domingues, J. P. T., Fonseca, L., Sampaio, P., \& Arezes, P. M. (2016). Integrated versus nonintegrated perspectives of auditors concerning the new ISO 9001 revision. In 2016 IEEE International Conference on Industrial Engineering and Engineering Management (IEEM) (p. 866-870). Bali: IEEE. https://doi.org/10.1109/IEEM.2016.7798000

International Organization for Standardization. (2018). The integrated use of management system standards. ISO Handbook ( $2^{\circ}$ ed). Genebra. Recuperado de https://www.iso.org/files/live/sites/isoorg/files/store/en/PUB100435_preview.pdf

Organização Internacional de Normalização (2019). Normas de sistema de gestão. Recuperado 19 de outubro de 2019, de https://www.iso.org/management-systemstandards.html

International Organization for Standardization. (2018). ISO/IEC 27000 - Information 
technology - information security management systems - overview and vocabulary. Genebra.

International Organization for Standardization. (2018). ISO 45001 - Sistemas de gestão da saúde e segurança ocupacional - requisitos com orientação para uso. Genebra.

Marinkovic, S. (2019). Lista de normas ISO de sistemas de gestão. Recuperado 19 de outubro de 2019, de http://isotc.iso.org/livelink/livelink?func=ll\&objId=18964860\&objAction=Open\&nexturl=\%2 Flivelink\%2Flivelink\%3Ffunc\%3D11\%26objId\%3D16474137\%26objAction\%3Dbrowse\%26 viewType\%3D1

Martín-Martín, A., Orduna-Malea, E., Thelwall, M., \& Delgado López-Cózar, E. (2018). Google Scholar, Web of Science, and Scopus: A systematic comparison of citations in 252 subject categories. Journal of Informetrics, 12(4), 1160-1177. https://doi.org/10.1016/j.joi.2018.09.002

Nowicki, P., Kafel, P., \& Sikora, T. (2013). Selected requirements of integrated management systemsbased on PAS 99 specification. International Journal for Quality Research, 7(1), 97106. Recuperado de http://www.ijqr.net/journal/v7-n1/7.pdf

Pardo, C., Pino, F. J., \& Garcia, F. (2016). Towards an Integrated Management System (IMS), harmonizing the ISO/IEC 27001 and ISO/IEC 20000-2 standards. International Journal of Software Engineering and Its Applications, 10(9), 217-230. https://doi.org/10.14257/ijseia.2016.10.9.18

Prodanov, C. C., \& Freitas, E. C. de. (2013). Metodologia do trabalho cientifico: métodos e técnicas de pesquisa e do trabalho acadêmico. Universidade FEEVALE ( $2^{\circ}$ ed). Novo Hamburgo: Feevale. Recuperado de http://www.feevale.br/Comum/midias/8807f05a-14d04d5b-b1ad-1538f3aef538/E-book Metodologia do Trabalho Cientifico.pdf

Vroegindeweij, R., \& Carvalho, A. (2019). Do Healthcare Workers Need Cognitive Computing Technologies? A Qualitative Study Involving IBM Watson and Dutch Professionals. Journal of the Midwest Association for Information Systems (JMWAIS), 2019(1), 51-68. https://doi.org/10.17705/3jmwa.000047 
Porcentagem de contribuição de cada autor no manuscrito

Diogo Pedriali - $60 \%$

Marilia Macorin de Azevedo - 15\%

Carlos Hideo Arima - 15\%

José Manoel Souza das Neves - 10\% 